\title{
El sistema de gestión de calidad: elemento para la competitividad y la sostenibilidad de la producción agropecuaria colombiana
}

\section{The quality management system: an element for the competitiveness} and sustainability of agricultural production in Colombia

Jorge Armando Fonseca C. ${ }^{1}$, Nelson Armando Muñóz P. ${ }^{2}$ \& José Alejandro Cleves L. ${ }^{3}$ jorge.fonseca@unad.edu.co,nelson.munoz@uptc.edu.co, clevesalejandro@yahoo.com

${ }^{1}$ Universidad Nacional Abierta y a Distancia (UNAD)

Escuela de Ciencias Agrícolas Pecuarias y del Medio Ambiente (ECAPMA)

CEAD Tunja, Colombia

Grupo Interinstitucional de Investigación en Gestión Ecoambiental y Sistemas Sostenibles de Producción (GIGASS)

UNAD - UPTC

${ }^{2}$ Universidad Pedagógica y Tecnológica de Colombia (UPTC)

Facultad de Estudios a Distancia

Tunja, Colombia

${ }^{3}$ Universidad Pedagógica y Tecnológica de Colombia (UPTC)

Escuela de Administración de Empresas Agropecuarias

Duitama, Colombia

Resumen.- En las condiciones de creciente globalización de los mercados, cambio en los hábitos de consumo, frecuente aparición de brotes de enfermedades transmitidas por alimentos (ETAS), las organizaciones sociales y los gobiernos buscan que la producción y provisión de alimentos incluya el aseguramiento de la inocuidad en todos los eslabones de la cadena. Una de las formas para lograrlo, es la implementación de un Sistema de Gestión de Calidad (SGC) con fines de certificación en las unidades productivas agropecuarias; el proceso aborda la finca desde la óptica de la teoría general de los sistemas, configurada por subsistemas productivos y de soporte; en ellos se desarrollan actividades tanto técnicas como administrativas que buscan optimizar los procesos, manteniendo criterios de sostenibilidad y responsabilidad social con el fin de lograr la producción de alimentos con criterios de calidad previamente establecidos. El proceso de desarrollo e implementación del SGC requiere la construcción y puesta en funcionamiento de documentos o manuales soporte en aspectos administrativos, productivos y de organización de productores, que están estructurados para responder a las exigencias de una norma, protocolo o código de conducta, su cumplimiento se verifica a través de auditorías que pueden llevar al otorgamiento de la certificación o sello de calidad del producto o servicio, el proceso se puede desarrollar de forma individual por un productor, o por una organización de productores.

Palabras clave: Alimentos, Enfermedades Trasmitidas por Alimentos (ETAS), Sistema de Gestión de Calidad (SGC), Inocuidad de alimentos, Certificación, Buenas Prácticas Agrícolas (BPA)

\section{Abstract}

Under the conditions of the increasing globalization of markets, changing consumer habits, frequent outbreaks of foodborne illnesses (FBI), social organizations and governments seeking production and food supply include the assurance of safeness in all links in the chain. One way to achieve it, is to implement a Quality Management System (QMS) for certification in agricultural production units, the process addresses the farm give the perspective of general systems theory, shaped by production subsystems and support; 
them develop both technical and management activities that seek to streamline processes while maintaining standards of sustainability and social responsibility to achieve food production with previously established quality criteria. The process of developing and implementing the QMS requires the construction and operation of support documents and manuals on issues of organization, and organization of producers, which are structured to meet the requirements of a standard, protocol or code of conduct; their compliance is verified through inspections that could lead to the granting of certification or seal of product quality or service, the process can be developed individually by a producer or a producer organization.

Keywords: foodborne illnesses, Quality Management System (QMS), Food safety, supply chain management, Certification, Good Agricultural Practices (GAP)

\section{Definiciones}

Aseguramiento de la inocuidad: conjunto de condiciones y medidas necesarias durante la producción, elaboración y almacenamiento de alimentos, así como su distribución y preparación, para asegurar que una vez ingeridos no representan un riesgo apreciable para la salud.

Auditoria: proceso sistemático, independiente y documentado para obtener evidencias de evaluación objetiva, con el fin de determinar en qué grado se cumplen los criterios exigidos.

Calidad: grado en el que en un conjunto de características inherentes cumple con los requisitos, bajo determinadas condiciones de uso.

Certificación de calidad: es el resultado de evaluar el cumplimiento de unos requisitos los cuales se comparan frente a unos referenciales definidos que pueden ser normas técnicas, reglamentos técnicos o especificaciones de clientes. Como resultado de lo anterior, se obtiene un certificado (documento donde se denuncia el cumplimiento de los requisitos). Esto se hace mediante el proceso que realiza un tercero que corresponde a un organismo de certificación el cual debe estar reconocido por una institución que para el caso de Colombia es la Superintendencia de Industria y Comercio quien da el aval para otorgar, mantener, extender y retirar la certificación.

Competitividad: es la capacidad de una organización de mantener sistemáticamente ventajas comparativas que le permitan alcanzar sostener y mejorar una determinada posición en el entorno socioeconómico.

Inocuidad: es la condición de los alimentos que garantiza que no causarán daño al consumidor cuando se preparen y/o consuman de acuerdo con el uso al que se destinan.

Manual de calidad: constituye un primer documento básico que enuncia una política de calidad global y describe el sistema de calidad de la organización, incluye distintas funciones como la de brindar indicaciones importantes a asesores externos. El manual también puede establecer un puente efectivo entre las actividades reales de la empresa y una norma de certificación a la que esté aplicando.

Procedimiento: manera específica de realizar una actividad. Contiene los propósitos y alcances de una actividad. Generalmente responde a preguntas: ¿Qué se debe hacer y quién lo debe realizar? ¿Cuándo, dónde y cómo se debe hacer? ¿Materiales, equipos y documentos que se deben usar? ¿Cómo se controlará y registrará dicha actividad?

Sistema de control interno: en su concepto más amplio puede cubrir todas las operaciones y actividades tendientes a mejorar el proceso productivo. Dentro de las características de este sistema es la descentralización operativa (a veces denominado control gerencial e incluyendo la auditoría interna), la centralización normativa y la centralización de la auditoría externa. 
Sistema de gestión de calidad: es un sistema basado en el sentido común, bien documentado, que asegura consistencia y mejoras en las prácticas de trabajo y en los productos y servicios que se crean. Se sustenta en normas que especifican un procedimiento para lograr una efectiva administración de calidad.

Trazabilidad: es el proceso mediante el cual se monitorean de manera permanente los eventos realizados en todo el proceso productivo con el objetivo de que el comprador tenga acceso a toda la información de su origen e historia del producto a consumir; a sí mismo, es la "habilidad de trazar el origen y la historia de transformaciones de una entidad por medio de registros de información", con el fin de generar confianza al consumidor del producto. Este proceso facilita la detección de errores a lo largo de la cadena de producción, transformación y distribución. La trazabilidad representa el rastreo de un producto desde un punto cualquiera de las cadenas hasta su origen.

\section{Siglas}

APPCC: Análisis de Peligros y Puntos Críticos de Control; en inglés HACCP (Hazard Analysis and Critical Control Points)

BPA: Buenas Prácticas Agrícolas; en inglés GAP (Good Agricultural Practices)

GLOBALGAP: Global Good Agricultural Practices; antes conocido EUREPGAP (Euro-Retailer Produce Working Group)

ETAS: Enfermedades Transmitidas por Alimentos

FAO: Organización de las Naciones Unidas para la Agricultura y la Alimentación (Food and Agriculture Organization of the United Nations) ISO: International Organization for Standardization

OHSAS: Serie de Normas de Evaluación en Seguridad Industrial y Salud (Occupational Health and Safety Assessment Series)

ONN: Organismos Nacionales de Normalización
PHVA: ciclo Deming o de control de calidad por sus iníciales: Planear, Hacer, Verificar y Actuar correctivamente

POES: Procedimientos Operativos Estandarizados

SGC: Sistema de Gestión de Calidad

\section{Introducción}

Los criterios de inocuidad y gestión de la calidad en la producción agropecuaria, han cobrado importancia en los últimos años, consumidores y gobiernos buscan la implementación de mecanismos para mejorar la producción y provisión alimentaria y lograr su aseguramiento. Lo anterior está sustentado en la frecuente aparición de enfermedades que tienen su origen o son transmitidas por los alimentos, comúnmente llamadas (ETAS). Las ETAS son responsables de un elevado número de eventos que incluyen muertes e intoxicaciones tanto en países importadores como productores de alimento para consumo en fresco o mínimamente procesados. Para contrarrestar o al menos mitigar sus impactos en la población, se han venido desarrollando e implementando mecanismos que permiten responder efectivamente a los requerimientos de los consumidores en términos de inocuidad y calidad mediante la regulación de los procesos productivos, de transformación y distribución (Arenas 2000). Lo anterior es posible lograrlo con la implementación de un SGC.

Este documento tiene como objetivo mostrar la importancia que para el sector hortofrutícola tiene la implementación del SGC como elemento fundamental en el proceso de aseguramiento de la inocuidad, la sostenibilidad ambiental y el bienestar de los trabajadores rurales y sus familias; además se muestran los principales componentes del sistema y cómo éstos se estructuran y aplican dentro de la unidad productiva para dar cumplimiento a una normativa, reglamento o código de conducta (FAO et al. 2001). 


\section{La inocuidad y las enfermedades transmitidas por alimentos ETAS}

La producción agropecuaria a nivel mundial desde hace varios años inició una serie de transformaciones que buscan adecuarla a los requerimientos de los consumidores y constantes cambios en la demanda con la preferencia de alimentos más sanos, de origen natural, funcionales y cuya producción sea amigable con el medio ambiente, sin descuidar las condiciones de vida de los trabajadores rurales y la de sus familias. Uno de los aspectos que ha cobrado gran importancia en la producción de alimentos para consumo en fresco o con bajo nivel de procesamiento es el criterio de inocuidad; la FAO la define como "Condición o garantía de que un alimento no causará perjuicio al consumidor cuando éste sea preparado o ingerido de acuerdo con su uso previsto" (FAO \& OMS 2006). Esta creciente preocupación de los consumidores y de los gobiernos para mejorar las condiciones de inocuidad de los alimentos está sustentada en la cada vez más frecuente aparición, y en el reporte de casos en los cuales las enfermedades tienen su origen o son transmitidas por alimentos frescos o que fueron incluso procesados con tratamientos térmicos (ICA 2008), lo que sugiere que han sido producidos, almacenados, procesados y transportados en condiciones inadecuadas o poco seguras (Fonseca \& Cleves 2009); basta sólo mencionar el reciente caso (mayo de 2011) de aparición de la bacteria Escherichia coli en Europa causando un inusitado número de víctimas mortales, así como lo fue el caso de las vacas locas en Europa en 2004. Otros casos de presencia de patógenos de alta virulencia que afectaron a segmentos de la población con cambios inmunológicos e intoxicaciones masivas en los Estados Unidos se registraron con la bacteria Salmonella saintpaul en tomates y melones en el año 2008; la presencia de Salmonella sp. en el interior de huevos frescos que causó el retiro del mercado de más de 500 millones de unidades por estar potencialmente contaminados con la bacteria; las frambuesas que contenían altas concentracio- nes de Cyclospora, provenientes de Guatemala y que causaron una intoxicación masiva no sólo en Estados Unidos sino en Europa; para el 2010 se reportaron más de 1.300 casos de intoxicaciones en Estados Unidos. En Colombia, en el 2002 se presentó una intoxicación masiva en Medellin asociada con un brote de Cyclospora cayetanensis presente en alimentos (Almirall et al. 2008, Botero-Garcés et al. 2006); la presencia en la leche para consumo humano de una alta concentración de peróxidos, sustancia química que se usa como desinfectante en equipos de pasterización.

Este tipo de eventos han impulsado a los gobiernos, las empresas y los productores organizados a desarrollar mecanismos que permitan responder efectivamente a la demanda de los consumidores, regulando los procesos de producción, transformación, distribución y comercialización que asegura la inocuidad de los alimentos. Para lo cual se requiere de una política de inocuidad alimentaria que reconozca las interrelaciones en los procesos de la cadena, de forma tal que se pueda proteger la salud de los consumidores a través de la implementación de mecanismos de monitoreo de peligros, valoración y mitigación de riesgos (Mateos 2002).

Para los fines del documento, el peligro, se define como un agente físico, químico o biológico presente en un alimento o bien, con la condición que éste pueda causar un efecto adverso para la salud de productores y consumidores; de la misma forma, el riesgo, es la posibilidad de ocurrencia o materialización de un peligro (FAO 2008).

El punto de partida para garantizar alimentos inocuos es el análisis de peligros, el cual consiste en la identificación minuciosa de los peligros físicos, químicos y biológicos que se puedan presentar, de acuerdo con las condiciones y las características del sistema de producción y del mismo producto. Una vez identificados esos peligros, se debe evaluar la importancia o riesgo potencial de cada uno, considerando su 
probabilidad de ocurrencia y gravedad (Codex alimentarius 2006). El sistema APPCC (HAC$\mathrm{CP}$ ) establece los criterios y lineamientos para el aseguramiento de la inocuidad en los procesos de producción alimentaria, principalmente a nivel industrial, donde se ha logrado un relativo avance. Para el sector agropecuario en general y para la producción primaria en particular se requiere para su exitosa aplicación la existencia de planes o programas pre-requisito de soporte funcionando dentro de la unidad productiva hortícola, entre las posibles herramientas que funcionan a manera de planes para la producción de alimentos inocuos están las Buenas Prácticas Agrícolas (BPA), Buenas Prácticas de Manufactura (BPM), Procedimientos Operativos Estandarizados (POES), entre otros (Fonseca \& Cleves 2009, Fedegan 2005).

\section{El Sistema de Gestión de la Calidad - SGC de la empresa agropecuaria}

Las Buenas Prácticas Agrícolas (BPA) para la producción primaria de alimentos, son el conjunto de criterios que involucran tanto aspectos relativos a la actividad agrícola como principios de buenas prácticas de manufactura y buenas prácticas de higiene, los cuales son transversales en la producción; esta unión de criterios constituyen un sistema de aseguramiento de la inocuidad, que con enfoque preventivo se aplica a toda la cadena, reuniendo las condiciones operativas adecuadas para el mejoramiento de los métodos convencionales de producción, haciendo énfasis en la inocuidad del producto, y con el menor impacto sobre el ambiente, la fauna, la flora y la salud de los trabajadores y sus familias (Contreras \& Restrepo 2007). Para el caso de la agricultura en Colombia, estos aspectos mencionados anteriormente han venido en creciente proceso de implementación o adopción por parte de las autoridades sanitarias, las empresas comercializadoras y en menor medida por los productores (Montañez 2009), lo que hace necesario que se intensifiquen las actividades para generar mecanismos que permitan a los pequeños y medianos pro- ductores hortícolas desarrollar en su unidad productiva un SGC para adoptar sin mayores traumatismos, bajo el enfoque administrativo, aspectos relacionados con la producción, inocuidad, calidad y sostenibilidad de la actividad agropecuaria (Fonseca \& Vega 2010).

Para tener un mayor nivel de comprensión del SGC, se propone descomponerlo en sus elementos constituyentes.

El primer elemento es el sistema partiendo de la teoría general de sistemas propuesta por Bertalanffly. Se define como un conjunto de partes o elementos relacionados y organizados que interactúan entre sí y con el ambiente, es decir actúan como un todo para lograr un objetivo o propósito y dar respuesta a una necesidad concreta (Bertalanffy 1976). Para abordar esta temática con los productores agrícolas, es necesario hacerlo desde la óptica de la finca o unidad productiva como el sistema Empresa Agropecuaria (Contreras \& Restrepo 2007), la cual tiene límites en términos geográficos (linderos) y en térmicos de su actividad productiva (lo que se encuentra fuera de estos linderos se considera como un suprasistema, ya que posee sus propios elementos constitutivos y su propia dinámica); igualmente el sistema finca o unidad productiva hortícola posee entradas en términos de energía, recursos y insumos que son básicos para el proceso productivo; unas salidas que son la resultante de la interacción de los insumos o entradas que están reportados en términos de productos biológicos, ambientales y financieros, que en últimas son la razón de ser del proceso productivo (Espejo 1996). Por lo que el inadecuado balance o manejo de los elementos que constituyen el sistema son una de las principales causas para reducir tanto la calidad, rentabilidad y sostenibilidad de la actividad agrícola.

Un segundo componente es el concepto de la gestión, entendiéndose ésta como "La administración de los recursos, la movilización de voluntades y la toma de decisiones, para la máxi- 
ma consecución de los objetivos con recursos escasos" (Simón citado por Contreras \& Puentes 2009). En términos de la empresa agropecuaria la gestión se puede entender como un conjunto de dimensiones que involucran estrategias y medios para alcanzar los objetivos en términos de productividad, rentabilidad y sostenibilidad de las actividades de la empresa agropecuaria (Linares \& Salamanca 2003); es sin duda la capacidad que ésta desarrolla para garantizar que su proceso productivo cumpla con las expectativas tanto de los clientes como de los inversionistas y trabajadores de la misma (Venegas 2006).

El tercer elemento que se debe considerar es la calidad en el contexto de la agroempresa, entendiéndose como el conjunto de propiedades y características de un producto o servicio que le confieren la aptitud para satisfacer necesidades expresas o implícitas (ISO 9000 versión 2004). Los aspectos de calidad hacen referencia a la calidad del producto (atributos físicos como color, aroma, sabor, textura, inocuidad, entre otros aspectos), también puede hacer referencia a la calidad en el servicio, que es el resultado generado por las actividades de la interfaz entre el proveedor y el cliente y por las actividades internas del proveedor con el fin de responder a las necesidades del cliente y la calidad del proceso. La norma ISO 8402 de 1994 define el proceso como "El conjunto de recursos y actividades relacionadas entre sí y que transforman elementos de entrada y elementos de salida”, desde la perspectiva de la teoría general de sistemas el proceso agroempresarial es el flujo y las interacciones de las actividades que ocurren entre la oferta ambiental, la biodiversidad, el personal, las finanzas, los equipos, las instalaciones, el conocimiento, las técnicas, la formación y los métodos de producción relacionados con el cumplimiento de los requisitos preestablecidos (Fonseca 2007).

Por lo tanto, la gestión de calidad en las explotaciones hortícolas es un conjunto de actividades que desde la dirección (administradores y propietarios de la unidad productiva) determinan la política de calidad, los objetivos y las responsabilidades que se llevan a cabo por medios como la planificación, el aseguramiento y el mejoramiento de la calidad (ISO 8402 de 1994). En las unidades productivas es necesario establecer la mencionada política de calidad, como el marco de acción regulatoria sobre el cual se van a desarrollar las actividades de producción, adecuación, comercialización, transformación, en otras, que le permiten establecer sus objetivos de forma clara y concisa, estructurar un proceso de planeación, la producción en forma documentada (Contreras \& Puentes 2009), entre otros elementos igualmente importantes.

La gestión de la calidad usa como herramienta central PHVA (Borrego 2009), su función es desarrollar una serie de actividades al interior de la agroempresa para prevenir las causas que originan problemas en las fases iniciales de un proceso de producción, reduciendo los errores y desperdicios, pero siempre teniendo como soporte las evidencias escritas que se deben realizar en los registros de las actividades administrativas, técnicas y operativas de la producción. Sin duda la implementación del SGC debe ser una decisión del administrador o propietario (gerencia) que requiere total compromiso de todo el personal que se involucra en el proceso, ya que sin su efectiva participación no es posible lograr niveles de rentabilidad, atributos de valor y calidad del producto. Igualmente en la producción de hortalizas y en general de alimentos se debe contar con un profundo conocimiento de las etapas de producción, exigencias del mercado (Compes 2001), lo cual implica haber realizado un proceso riguroso de planeación de actividades que involucre y garantice la aplicación de criterios de inocuidad y calidad, a partir de BPA, Buenas Prácticas de Manufactura (BPM) y Buenas Prácticas de Higiene (BPH) (Fernández-Sierra 2003, Fonseca 2010).

La materialización del SGC en la agroempresa se inicia cuando se crean o diseñan los diferentes componentes para que éste pueda operar de 
forma adecuada, es decir el componente documental que ayuda al proceso de implementación, uno de los documentos de mayor importancia es el Manual de Calidad (MC), el cual se constituye en el documento básico donde la agroempresa enuncia el compromiso con la calidad de sus productos, $y$ en general describe el sistema de calidad (Contreras 2008). El Manual tiene distintas funciones incluyendo la de brindar indicaciones a los asesores externos, así como establecer los lineamientos entre las actividades de la producción con los criterios de calidad a los que se está aspirando llegar (Coronel 2007), los cuales pueden ser normas o códigos de cumplimiento a los que aspire la agroempresa con fines de certificación, el MC enuncia entre otros elementos, el funcionamiento de la organización, la política de calidad, su estructura administrativa, los objetivos y alcances, responsabilidades, funciones de sus integrantes y procesos administrativos; es la columna vertebral del SGC ya que permite a los trabajadores de la agroempresa entender y dimensionar las reales intensiones en términos de calidad y competitividad (Fonseca \& Vega 2010).

Un segundo documento que se usa como elemento fundamental para la implementación del SGC es el Manual de Procedimientos (MP). Este contiene e instruye sobre la forma en que se instrumentará o aplicará la política de calidad en las diferentes áreas que componen la agroempresa y constituye la guía de cómo se deben llevar a cabo las diferentes actividades por parte del personal tanto técnico, administrativo y de producción. Los procedimientos deben estar en forma documentada, es decir, escritos $\mathrm{y}$ al alcance de aquellos que necesitan aplicar o realizar dichos procedimientos (actividades de campo), debe contener como elementos mínimos los propósitos y el alcance de la actividad específica; la descripción detallada de cómo se debe proceder para realizarla en forma correcta en términos del cultivo, y segura para el operario que la realiza, las herramientas que se deben usar, las restricciones que se tienen en términos ambientales (conservación de oferta ambien- tal); y la forma como se debe registrar para garantizar el proceso de trazabilidad del producto (MADR \& Corpoica 2007). El MP debe poseer como mínimo tres secciones a saber: 1) procedimientos administrativos: fundamentalmente describe el proceso mediante el cual se puede incorporar personal a las diferentes áreas de trabajo de acuerdo con la política de calidad ya mencionada, su forma de vinculación, capacitación, etc. 2) procedimientos operativos: hace referencia a la forma como la agroempresa desarrollará los procedimientos de compras, actividades de producción (las cuales se especificarán y describirán en forma detallada) y ventas. $3)$ procedimientos de soporte: éstos hacen referencia a cómo debe proceder la agrempresa ante eventualidades propias que surjan de los procesos de producción y comercialización, así como aspectos relacionados con incumplimientos y verificación de criterios de calidad e inocuidad, se sugiere que éste contenga procedimiento para reclamaciones, devoluciones, control de documentos y procedimiento para inspecciones internas (Contreras \& Restrepo 2007).

Un tercer documento que se considera importante en la agroempresa que implementa el SGC, es el Manual de Documentación (MD), éste contiene el listado de los documentos que se usan dentro del SGC con sus respectivos códigos (los cuales obedecen al proceso de sistematización interno), además de poseer los documentos o formas sin diligenciar (formatos) que se requieren para consignar la información de las actividades realizadas (registros) con los que se construye y configura la trazabilidad del producto. Para estructurar la documentación de todo sistema de gestión es necesario tener en cuenta los requisitos que contempla la norma ISO 9001 (Montañez 2009).

\section{Normas, protocolos y códigos de conducta}

Se puede afirmar que el marco normativo a partir del cual se estructura la gestión de calidad en el sector agropecuario es la familia de normas 
ISO sobre la cual se han construido la mayoría de los protocolos y códigos de conducta para este sector. Entre las más importantes y que contribuyen a la estructuración de las normativas de calidad está ISO 9000 la cual describe los fundamentos de los sistemas de gestión de la calidad y la terminología usada; ISO 9001 especifica los requisitos para los sistemas de gestión de la calidad aplicables a toda organización que decida iniciar procesos de mejoramiento, generalmente con la intención de cumplir requerimientos de mercados especializados; ISO 9004 proporciona directrices que consideran tanto la eficacia como la eficiencia del sistema de gestión de calidad, el objetivo de esta norma es mejorar el desempeño de la organización y la satisfacción de los clientes; ISO 19011 proporciona orientación relativa a las auditorias de sistemas de gestión de calidad y de gestión ambiental. A esta amplia familia se le adicionan también el grupo de normas ISO 14000 las cuales se encargan de regular los criterios de cumplimiento de la gestión ambiental, que para el sector agropecuario se ha convertido en un elemento imprescindible en los crecientes mercados especializados. Además de las anteriores, se tienen en cuenta los criterios de ISO 18000 o de las normas conocidas como OHSAS que establecen los aspectos de seguridad y salud para los trabajadores, en este caso se interpretan de acuerdo a las actividades y niveles de riesgo de las labores agrícolas. Estos criterios anteriormente citados se enfocan y se contextualizan desde la óptica del Codex Alimentarius desarrollado por el comité del Codex adscrito a la FAO y a la organización mundial del comercio OMC bajo el auspicio de la ONU. El Comité es el responsable de la elaboración de normas y códigos de prácticas de ámbito mundial para productos alimentarios, tanto así que se considera el referente normativo de estos aspectos. En igual sentido ISO 22000 define los requisitos relativos a la gestión de la inocuidad alimentaria, constituyendo un estándar que armoniza las obligaciones de productores con las necesidades y exigencias de los mercado y los consumidores, la norma puede ser aplicada por cualquiera de las organizaciones implicadas en la cadena alimentaria, desde las unidades productivas hasta las empresas agroindustriales (Nancucheo et al. 2004), incluyendo las de procesado, envasado, transporte, almacenamiento y comercio. Como se mencionó, las normas que aplican al sector agropecuario están casi en su totalidad basadas en la articulación de los principios normativos anteriormente señalados, y difieren del enfoque o incluso del producto.

Para el sector hortofrutícola colombiano las principales normas, protocolos y códigos de conducta que se están implementando con fines de certificación, principalmente para procesos de exportación, se pueden dividir en cuatro grandes grupos 1) basadas en BPA. 2) Producción orgánica; 3) ambientales 4) de responsabilidad social. Al primer grupo pertenece la norma Globalgap que hasta el año 2007 se denominó Eurepgap desarrollada por un grupo de supermercados en Europa (Oyarzún \& Tartanac 2002), y que se constituye en requisito de obligatorio cumplimiento para las exportaciones agropecuarias hacia la Unión Europea y Estados Unidos (Torrado 2005). Para cultivos como café y cacao existe el código de conducta UTZ Certified (anteriormente denominada Utz kapeh). Este código es un conjunto de criterios sociales, ambientales y técnicos reconocidos internacionalmente para la producción responsable, se basa en los principios establecidos por la Organización Internacional del Trabajo (OIT) y Globalgap (Cáceres et al. 2004); la NTC 5400 regulada por la resolución 4174 de 2009 del Instituto Colombiano Agropecuario - ICA "Buenas prácticas agrícolas para frutas, hierbas aromáticas culinarias y hortalizas frescas", proporciona los requisitos generales y las recomendaciones para el contexto colombiano, a la vez que se trabaja en un proceso de homologación con Globalgap (Globalgap 2007), que permita obtener el estándar Colombiagap.

En el grupo de normas ambientales existe un amplio número, las más conocidas son Rain- 
forest o Bird Friendly que establecen criterios de cumplimiento en términos de conservación ambiental para la producción agrícola (Oyarzun \& Tartanac 2002). En las normas para productos orgánicos las de mayor aplicación en el país es USDA NOP la cual es el parámetro para agricultura orgánica de los Estados Unidos (Pérez 2008) y es exigido en productos con destino a esa nación; El reglamento 889 y 834 (remplazó a la CEE 2092/91) es la norma de producción orgánica de Europa y aplica para los países que desean exportar alimentos ecológicos a la Unión Europea. La norma JAS aplica para exportar alimentos orgánicos al Japón. Es importante aclarar que cada norma tiene diversos puntos de control y criterios de cumplimiento, así como diferentes niveles de exigencia; la norma colombiana de producción

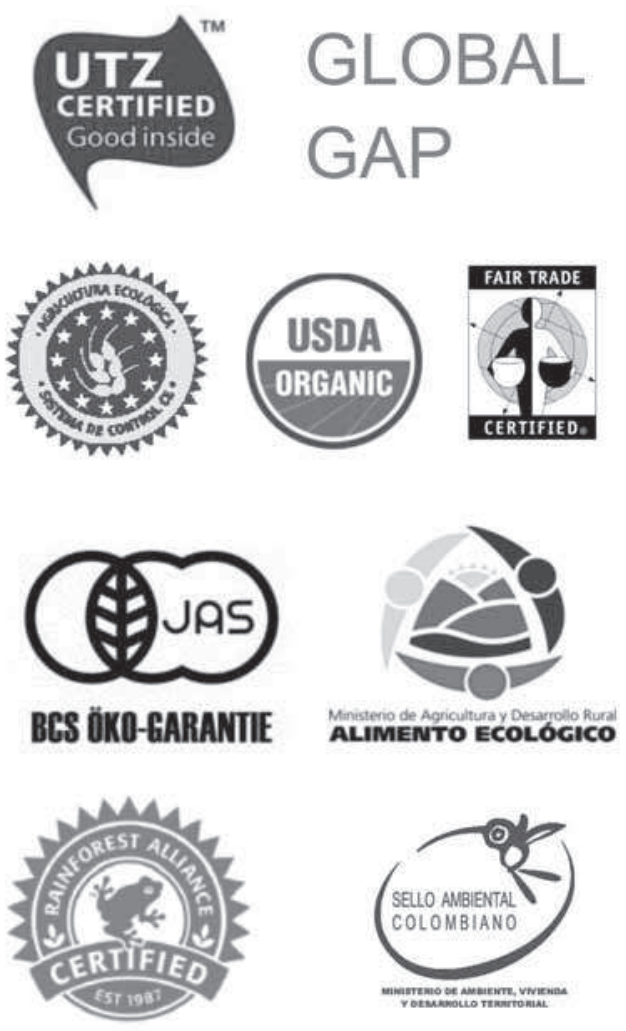

Figura 1. Principales sellos de calidad del sector agropecuario. ecológica es la Resolución 0187 de 2006, define los parámetros para agricultura ecológica en el país. Para el cuarto y último grupo propuesto, están las normas de responsabilidad social como la certificación Fair Trade o de comercio justo para el sector agropecuario (Figura 1).

\section{El proceso de implementación con fines de certificación}

Cuando las unidades productivas agrícolas en forma individual o forma conjunta con otros productores están dispuestos a desarrollar e implementar el SGC y deciden dar cumplimiento a una norma o protocolo de acuerdo a las exigencias de los compradores o al interés en un mercado determinado. Este proceso debe ser inspeccionado y aprobado por una entidad independiente y especializada, que para el caso son las empresas certificadoras (CAPLAM 2006), dando inicio al proceso de implementación con fines de certificación, entendiendo ésta como el resultado de evaluar el cumplimiento de los requisitos especificados los cuales se comparan frente a referentes definidos como son las normas técnicas, reglamentos, protocolos, o códigos de conducta.

El consumidor al adquirir un alimento de origen agrícola busca que éste tenga características que satisfagan sus necesidades o intereses particulares en términos de calidad e inocuidad; ésta decisión de compra necesita elementos que le ayuden a generar o mantener la confianza sobre los alimentos que adquiere y sobre su productor/proveedor (FernándezSierra 2003), de tal forma que pueda confiar en la información de la etiqueta, saber el origen, sus características, la forma de producción, etc. Por lo tanto los consumidores requieren de un sistema confiable que les brinde seguridad en la relación productor - consumidor (FLO 2002). En ese sentido la Certificación Agropecuaria aparece como un mecanismo que sirve para identificar el producto y asociarlo con sus características especificas en cuanto a parámetros de calidad. La certificación es por tanto una 
confirmación de manera formal y escrita que una entidad especializada otorga al productor o grupo de ellos en la constancia de su cumplimiento a la norma o especificación voluntaria con la cual se habían comprometido (IFAT 2002). La certificación entonces se convierte en un elemento que permite la confianza y facilita la identificación de los productores. Este proceso se realiza a través de un proceso de auditoría o verificación por parte del ente certificador a los procesos administrativos, técnicos y de producción de las unidades en forma individual o grupal (Hernández 2009), cuando se presenta esta circunstancia de grupo de productores se desarrolla e implementa el SCI que constituye el reglamento máximo (las reglas de juego) a través del cual el grupo de productores se interrelacionan entre sí, con los proveedores y los clientes (Contreras \& Puentes 2009).

\section{Sistemas de certificación}

Con la globalización de la economía mundial y el aumento en los volúmenes del comercio internacional, el cual se ha incrementado principalmente por la liberalización de la economía mundial y la eliminación de algunas barreras al comercio, la importancia de la normalización ha aumentado y con él la relevancia de ONN (Montañez 2009).

En el pasado los ONN, eran instituciones gubernamentales o privadas, diseñadas de alguna manera para proteger a la industria nacional, con normas hechas localmente y a la medida de cada país (Correa 2009). El papel de los ONN era reducido en cuanto a su alcance, ya que las normas no tenían un destacado papel en el desarrollo de la economía del país y el mercado local estaba cerrado a la competencia externa. En el mundo globalizado, las normas han adquirido una gran importancia como instrumentos para evitar las barreras no arancelarias (o para arancelarias) y para buscar una mayor compatibilidad que facilite el comercio a través de las fronteras. De esta forma, el liderazgo en normalización, certificación y calidad, ayuda a la industria local a incrementar su competitividad, contribuyendo al posicionamiento de los sectores industriales y de servicios del país en el contexto internacional (Arenas 2000).

La normalización es un instrumento de ordenamiento tecnológico que ofrece a la sociedad importantes beneficios, al facilitar la adaptación de los productos, procesos y servicios a los fines a los que se destinan, protegiendo la salud y el medio ambiente, previniendo los obstáculos al comercio y facilitando la cooperación tecnológica (Torrado 2005).

La actividad de certificación ha cobrado más importancia desde la aprobación y aceptación de ISO 9000 y sus similares, y la necesidad de las industrias locales de un reconocimiento fuera de sus límites fronterizos; instrumento imprescindible para elevar el nivel de calidad de los productos, los servicios y las empresas de un país. La certificación de un producto también se lleva a cabo en gran medida y es utilizada, especialmente a través de acuerdos comerciales bilaterales (Fonseca \& Vega 2010).

La certificación es entonces, la acción llevada a cabo por una entidad reconocida como independiente de las partes interesadas, que da fe y lo manifiesta por escrito, que un producto, proceso o servicio debidamente identificado es conforme con una norma o documento normativo especificado; en virtud de una verificación de sus propiedades y características, que están de acuerdo con las normas y especificaciones técnicas. La certificación es actualmente una herramienta ineludible, que facilita a las empresas la introducción de sus productos y servicios en otros mercados. Se trata en definitiva de un procedimiento de aseguramiento de la conformidad que resulta en una declaración escrita (licencia o certificado) que da la confianza de que el objeto de certificación satisface los requisitos establecidos (Fernández-Sierra 2003). Así mismo, se establece un sistema de sello o marca de conformidad que se imprime en cada producto para atestiguar que satisface los requisitos esta- 
blecidos en la norma de aplicación respectiva. Este sistema de marca posee un reglamento de contratación y uso, acompañado de un proceso que comprende las siguientes acciones:

- Concesión de la marca, tras el estudio de la documentación técnica, identificación de la norma de aplicación, análisis de las muestras iniciales, ensayos de prototipos, aprobación y firma del contrato.

- Auditoria del producto, mediante la comprobación periódica y control del mantenimiento de las características aprobadas en los prototipos. Este proceso de seguimiento periódico de la continuidad del sistema de calidad, también comprende la recepción y verificación de denuncias de clientes o competidores (ISO 9011, 2000).

- Sanción de infracciones, que puede llevar a la suspensión temporal del sello o el retiro definitivo del certificado.

Este sistema de certificación puede referirse al cumplimiento de las condiciones de rendimiento y seguridad; o bien puede considerar solamente las condiciones de seguridad del producto, diferenciándose claramente el tipo de sello a brindar en cada caso (Fonseca \& Cleves 2009).

\section{Conclusiones}

Los cambios en las dinámicas de producción y comercialización de alimentos a nivel global, y los diferentes hábitos de consumo de amplios sectores de la población que exigen alimentos frescos y naturales, han permitido que en primera instancia los productos agropecuarios y los patógenos asociados estén presentes en forma generalizada a nivel mundial sin fronteras geográficas o de condición social, condición que han propiciado la creciente aparición de brotes de enfermedades trasmitidas por alimentos.

En los últimos años la preocupación de la inocuidad alimentaria ha cobrado importan- te espacio en las políticas de desarrollo de los países, tanto productores como compradores, incidiendo en la promoción de actividades regulatorias de los procesos de producción, transformación y distribución de alimentos. Para el caso del sector hortícola nacional se viene desarrollando un proceso de implementación de un SGC que le permita aumentar su competitividad y penetrar en otros mercados.

Para lograr estas premisas el sector hortícola deberá seguir en su proceso de reconversión a partir de la implementación de tecnologías de bajo costo, que aumenten la productividad, y que sean respetuosas al medio ambiente; estos conceptos estarán directamente relacionados con la implementación del SGC en cumplimiento a los protocolos, normas y códigos de conducta que exigen los nuevos mercados.

\section{Literatura citada}

Abarca, R. \& S. Sepúlveda. 2001. Eco-etiquetado: un instrumento para diferenciar productos e incentivar la competitividad. Cuaderno Técnico No. 17. IICA; Coronado, Costa Rica.

Almirall, P., A. Escobedo \& S. Cimerman. 2008. Cyclospora cayetanensis un protozoo intestinal emergente. Revista Panamericana de Infectología 10: 24-29.

Arenas, A. 2000. El aseguramiento de la calidad e inocuidad de los alimentos. Editorial Retina, Bogotá.

Borrego, D. 2009. Herramientas para la mejora continua, Ciclo Deming. Blog Herramients para Pymes. Disponible en línea: http:/ / www.herramientasparapymes.com/herramienta-para-lamejora-continua-ciclo-deming (Consultado en julio de 2011).

Botero-Garcés, J., M.N. Montoya-Palacio, J.I. Barguil \& A. Castaño-González. 2006. Brote epidémico por Cyclospora cayetanensis en Medellín Colombia. Revista de Salud Pública 8: 258-268. 
Buxade, C. 1999. Enciclopedia práctica de la agricultura y la ganadería. Editorial Océano, Barcelona, España.

Cáceres, F., T. Manrique, T. Parra \& J.C. Cruz. 2004. Nuevos Sistemas Agrarios y su posicionamiento en la cadena agroalimentaria. Informe de la Agricultura Familiar en España. Fundación de Estudios Rurales. Madrid

Codex Alimentarius. 2006. Programa conjunto sobre normas alimentarias. Comisión del codex alimentarius. FAO/OMS. Disponible en línea: http://www.codexalimentarius.net/download/ al29_22s.pdf [consultado en julio de 2011].

Comisión Latinoamericana de Productividad y Medio Ambiente (CAPLAM). 2006. Metodología para la certificación ISO 9001:2000 de empresas. Disponible en línea: http://www.clapam. com/paginas/4asesoria_certificacion.htm [consultado en julio de 2011].

Compes, R. 2001. Atributos de confianza, normas y certificación: comparación de estándares para hortalizas. Infoagro. Disponible en línea: http://www.infoagro.com [consultado en julio de 2011].

Contreras, U. 2008. El sistema de gestión de la calidad en la empresa agropecuaria. Editorial Ecopange Ltda, Bogotá.

Contreras, A. \& D. Puentes. 2009. Diseño del sistema de certificación de fincas ovinas en la provincia norte del departamento de Boyacá [Tesis Administrador de Empresas Agropecuarias]. Duitama: Universidad Pedagógica y Tecnológica de Colombia.

Contreras, U. \& S. Restrepo. 2007. Manual de implementación del sistema de control interno como herramienta indispensable para el sector agropecuario. Editorial Ecopangea Ltda, Bogotá.

Coronel C., O.J. 2007. Manual para el manejo de ganado ovino. Telecentros Rura- les PAllasca Ancash. Disponible en línea: http://pallasca.inictel.net/img_upload/ a557d789319776f57046479435ef331f/MANUAL_PR_CTICO_GANADO_OVINO.pdf [consultado en julio de 2011].

Correa C., H.J. 2009. Normatividad en la producción agropecuaria en Colombia: aspectos generales. Universidad Nacional de Colombia. Disponible en línea: http://www.agro.unalmed. edu.co/departamentos/panimal/docs/Normatividad.pdf [consultado en julio de 2011].

Espejo M., C. 1996. Sistema de explotación ganadera: notas en torno a su concepto. Lurralde: investigación y espacio 19: 89-104.

FAO. 2008. Portal Internacional sobre Inocuidad de los Alimentos y Sanidad Animal y Vegetal. Disponible en línea: http://www.ipfsaph.org/servlet/ CDSServlet?status $=$ ND 1 jdGh0dHB3d $3 \mathrm{dmYW}$ 9vcmdhb3NoczAxMDQmNj1lcyYzMz0qJjM3P Wtvcw\%7E\%7E [consultado en julio 2010].

FAO \& OMS. 2006. Qué es el Codex Alimentarius. 3a Ed. Roma.

FAO; CCI \& CTA, 2001. Los mercados mundiales de frutas y verduras orgánicas. Oportunidades para los países en desarrollo en cuanto a la producción y exportación de productos hortícolas orgánicos. Roma.

Federación Colombiana de Ganaderos (Fedegan). 2005. Cartilla de registros de información para empresas ganaderas. Bogotá.

Fernández-Sierra, L.M. 2003. Las certificaciones de calidad. Análisis de riesgos y control de puntos críticos. En: Álvarez, J. y F. Camacho (Coord.). Innovaciones en el sector hortofrutícola español. Ministerio de Agricultura, Pesca y Alimentación, Madrid. pp. 95-113.

FLO. 2002. Overview of Fairtrade certified associations and plantations per country. Fairtrade Labelling Organizations International. Disponible 
en línea: http://www,fairtrade.net/ [consultado en Julio de 2011].

Fonseca, J. \& C. Vega. 2010. Diseño del sistema de gestión de calidad para empresas ovinocultoras en el departamento de Boyacá. En: Segundo Seminario Nacional y Primero Internacional en Salud y Producción Animal. Universidad Pedagógica y Tecnológica de Colombia (UPTC). Tunja, Colombia.

Fonseca C., J.A. 2010. Manual técnico del cultivo de cebolla puerro (Allium porrum) bajo enfoque de buenas prácticas agrícolas en el distrito de riego del alto Chicamocha. Orión Editores, Bogotá.

Fonseca, J. \& A. Cleves. 2009. Los sistemas de gestión de la calidad en la producción de ajo y cebolla en Colombia. En: Miranda L., M., C. Carranza, S. Magnitskiy, M. Rodríguez \& G. Fischer (Eds.). Memorias Tercer Congreso Colombiano de Horticultura y Simposio Internacional de Cebolla y Ajo en el Trópico. Sociedad Colombiana de Ciencias Hortícolas. Paipa, Colombia. p. 174

Fonseca, J. 2007. Contribución al análisis del estado de desarrollo de microempresas agroindustriales del sector lácteo en el corredor central de Boyacá 2007 [Tesis de grado Maestría en Desarrollo Empresarial Agropecuario]. Bogotá: Facultad de Agronomía de la Universidad Nacional de Colombia.

Globalgap. 2007 Reglamento general aseguramiento integrado de fincas. Parte III: certificación de grupo de productores. Código de Referencia: IFA 3.0-1 GR III. Versión: V3.0-1July07. Köln, Alemania.

Hernández, L. 2009. Certificación Agropecuaria. CIAT. Disponible en línea: http://www.ciat. cgiar.org/agroempresas/pdf/certificación. [consultado en julio de 2011].

Instituto Colombiano Agropecuario ICA. 2008. Inocuidad de los alimentos en la producción ganadera. Editorial Produmedios, Bogotá.
International Federation for Alternative Trade (IFAT). 2002. Estándares para las organizaciones de comercio justo. Disponible en línea: http://www.fongdcam.org/manuales/comerciojusto/datos/docs/ARTICULOS\%20Y\%20 DOCUMENTOS\%20DE\%20REFERENCIA\%20def/CONCEPTO\%20CJ/Definicion $\% 20 y \% 20$ contexto/7-EstandaresIFAT.pdf [consulta en julio de 2011].

International Organization for Standarization (ISO). 2004. ISO 9000:2004 Quality management systems. Fundamentals and vocabulary. 2nd Ed. Ginebra, Suiza.

International Organization for Standarization (ISO). 2000. ISO 9000:2000 Quality management systems. Fundamentals and vocabulary. 2nd Ed. Ginebra, Suiza.

International Organization for Standarization (ISO). 2000. ISO 9001:2000 Quality management systems. Fundamentals and vocabulary. Ginebra, Suiza.

International Organization for Standarization (ISO). 2002. ISO 19011:2000 Guidelines for quality and/or environmental management systems auditing. Ginebra, Suiza.

Linares, N. \& Y. Salamanca. 2003. Determinación e la capacidad de interacción de las empresas en un ambiente competitivo caso: empresas biotecnológicas [Tesis de grado Administradora de Empresa]. Bogotá: Facultad de Ciencias Económicas y Administrativas de la Universidad Nacional de Colombia.

Mateos, M. 2002. Seguridad e higiene en el sector de frutas y hortalizas. Temas de Actualidad No. 3. Instituto Tecnológico Agroalimentario (AINIA), Valencia, España.

Ministerio de Agricultura y Desarrollo Rural (MADR) \& Corporación Colombiana de Investigación Agropecuaria (Corpoica). 2007. Curso virtual guía para la implementación de las bue- 
nas prácticas ganaderas BPG's. Disponible en línea: http://www.corpoica.org.co/sitioweb/ Elearning/BPG/index.html [Consultado en julio de

Montañez, S. 2009. Diseño del sistema de certificación de conformidad de producto para BCS Oko garantie [Tesis de grado Administrador de Empresas Agraopecuarias]. Duitama: Universidad Pedagógica y Tecnológica de Colombia.

Ñancucheo, A., F. Rodríguez, A. Mansilla \& J.S Magaldi. 2004. Optimización de la cadena productiva y comercial con certificación de calidad. Revista IDIA 11: 170-172.

Oyarzún, M. \& F. Tartanac. 2002. Estudio sobre los principales tipos de sellos de calidad en alimentos a nivel mundial. Estado actual y perspectivas de los sellos de calidad en productos alimenticios de la agroindustria rural en América Latina. FAO, Santiago.
Pérez, L. 2008. Actualización - NOP. OCIA International, Inc. Disponible en línea: http://www. ocia.org/ResourceCenter/Training/NOP SP/7_NOP_Actualizacion_Final.pdf [consultado en julio de 2011].

Torrado, A. 2005. Buenas prácticas agrícolas. Sistema de aseguramiento de la inocuidad de los alimentos. Boletín Técnico. Instituto Colombiano Agropecuario. Bogotá.

Venegas, R. 2006. Buenas prácticas pecuarias. Asociación Gremial de Plantas Faenadoras Frigoríficas de Carnes de Chile. Disponible en línea: http://www.faenacar.cl/UserFiles/File/presentaciones/Rio\%20Verde\%202006/4RaulVenegasProducciónLimpia.pdf [consultado en julio de 201].

Von Bertalanffy, L. 1976. Teoría general de sistemas. Petrópolis, Vozes.

Recibido: 08 de abril de 2011 Aceptado: 15 de junio de 2011 